\title{
Blood Glucose Variability and Outcomes in Critically III Children
}

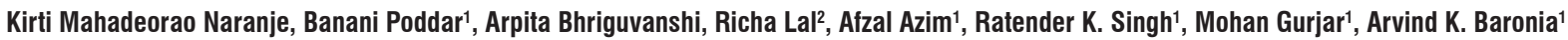

Departments of Neonatology, ${ }^{1}$ Critical Care Medicine and ${ }^{2}$ Paediatric Surgery, Sanjay Gandhi Postgraduate Institute of Medical Sciences, Lucknow, Uttar Pradesh, India

\section{Abstract}

Objectives: To find the incidence of hyperglycemia (blood glucose $[\mathrm{BG}] \geq 150 \mathrm{mg} / \mathrm{dl}$ ), hypoglycemia (BG $\leq 60 \mathrm{mg} / \mathrm{dl}$ ), and variability (presence of hypoglycemia and hyperglycemia) in critically ill children in the $1^{\text {st }}$ week of Intensive Care Unit (ICU) stay and their association with mortality, length of ICU stay, and organ dysfunction. Materials and Methods: The design was a retrospective observational cohort study. Consecutive children $\leq 18$ years of age admitted from March 2003 to April 2012 in a combined adult and pediatric closed ICU. Relevant data were collected from chart review and hospital database. Results: Out of 258 patients included, isolated hyperglycemia was seen in 139 (53.9\%) and was unrelated to mortality and morbidity. Isolated variability in BG was noted in 76 (29.5\%) patients and hypoglycemia was seen in $9(3.5 \%)$ patients. BG variability was independently associated with multiorgan dysfunction syndrome on multivariate analysis (adjusted odds ratio [OR]: 7.1; 95\% confidence interval [CI]: 1.6-31.1). Those with BG variability had longer ICU stay (11 days vs. 4 days, on log-rank test, $P=0.001$ ). Insulin use was associated with the occurrence of variability (adjusted OR: 3.6; 95\% CI: 1.8-7.0). Conclusion: Glucose disorders were frequently observed in critically ill children. BG variability was associated with multiorgan dysfunction and increased ICU stay.

Keywords: Blood glucose abnormalities, hyperglycemia, mortality, multiorgan dysfunction syndrome, variability

\section{INTRODUCTION}

Disruptions in normal homeostasis of blood glucose (BG) are common in critically sick children with unfavorable outcomes. While the adverse effects of hypoglycemia have been well documented in the pediatric literature, ${ }^{[1-3]}$ it was not until the landmark study by Van den Berghe et al. that controlling hyperglycemia with insulin was found to be associated with improved outcomes in critically ill patients. ${ }^{[4]}$ Subsequent studies in sick children also document adverse outcomes associated with hyperglycemia; ${ }^{[5-10]}$ however, recent randomized controlled trials of "tight glycemic control" (80-110 mg/dl;; ${ }^{[1]]} 72-126 \mathrm{mg} / \mathrm{d}\left[{ }^{[12]}\right)$ did not yield spectacular results. Lately, variability in BG levels has attracted much attention. Studies evaluating variability have shown increased adverse outcomes in critically ill adults. ${ }^{[13-15]}$ Similar literature in children is scarce. ${ }^{[8,10,16]}$

Literature from pediatric critical care evaluating glucose disorders and outcomes is limited. Available studies are from the developed world, and only a few are reported from resource-limited countries like India. ${ }^{[10,17]}$ Since the case mix and nutritional status are considerably different in our country, it is important to study the glucose abnormalities in our patient population. We, therefore, studied glucose disorders

\begin{tabular}{|l|l|}
\hline \multicolumn{3}{|c|}{ Access this article online } \\
\hline Quick Response Code: & Website: \\
& www.ijccm.org \\
\hline
\end{tabular}

in critically ill children and their relationship with mortality and morbidity.

\section{Materials and Methods}

This was a retrospective observational cohort study of children admitted to the Intensive Care Unit (ICU) of a tertiary care hospital in North India from March 2003 to April 2012. Ours is a 12-bedded combined adult and pediatric closed ICU for medical and surgical patients. All patients were under the direct supervision of critical care physicians (including one pediatric intensivist). The Institute's Ethics Committee approved the study with waiver of consent.

\section{Inclusion criteria}

All children $\leq 18$ years of age admitted in ICU who had at least two BG measurements during the first 7 days of ICU stay were included in the study.

Address for correspondence: Dr. Banani Poddar, Department of Critical Care Medicine, Sanjay Gandhi Postgraduate Institute of Medical Sciences, Lucknow - 226 014, Uttar Pradesh, India. E-mail: bananip@hotmail.com

This is an open access article distributed under the terms of the Creative Commons Attribution-NonCommercial-ShareAlike 3.0 License, which allows others to remix, tweak, and build upon the work non-commercially, as long as the author is credited and the new creations are licensed under the identical terms.

For reprints contact: reprints@medknow.com

How to cite this article: Naranje KM, Poddar B, Bhriguvanshi A, Lal R, Azim A, Singh RK, et al. Blood glucose variability and outcomes in critically ill children. Indian J Crit Care Med 2017;21:122-6. 


\section{Exclusion criteria}

Children with a diagnosis of diabetes mellitus, suspected or proven inborn errors of metabolism, ICU stay $\leq 12 \mathrm{~h}$, and incomplete medical records were excluded from the study.

\section{Measurements}

Data were retrieved from patient records and included demographic characteristics such as age, gender, weight, diagnosis, and medical or surgical admission. Maximum and minimum BG levels, presence of infection at admission, severity of illness, insulin and steroid usage, use of vasoactive agents, and mechanical ventilation in the first 7 days of ICU stay and outcomes were noted. BG levels were measured using point of care glucometer. Those with abnormal BG levels were more likely to have frequent BG assessment. In general, insulin infusion was started when BG levels were more than $200 \mathrm{mg} / \mathrm{dl}$ and titrated according to BG levels. The infusion was stopped when it was below $150 \mathrm{mg} / \mathrm{dl}$.

\section{Outcomes}

The primary outcome of the study was mortality at ICU discharge. Our secondary outcomes included length of ICU stay and multiorgan dysfunction syndrome (MODS).

\section{Definitions}

We took a BG cutoff of $150 \mathrm{mg} / \mathrm{dl}$ to define hyperglycemia. Hypoglycemia was defined as BG level $\leq 60 \mathrm{mg} / \mathrm{dl}$. BG variability was defined as the occurrence of both hypoglycemia and hyperglycemia in the first 7 days of ICU stay. ${ }^{[8,10]}$ The severity of illness at admission was assessed using Pediatric Index of Mortality-2 scoring system (PIM2). MODS was defined as the involvement of two or more organ systems. Patients were categorized into four mutually exclusive groups based on their BG values in the first 7 days: (a) hyperglycemia group, (b) hypoglycemia group, (c) normoglycemia group, and (d) variability group, which included one or more hypoglycemic and hyperglycemic values.

\section{Statistical analysis}

Data were analyzed using the statistical software SPSS 16.0 (SPSS, Chicago, IL, USA). Z-scores for weight for age were calculated using Epi Info software (Centers for Disease Control and Prevention, Atlanta, GA, USA). Nonparametric tests were used for comparison between the groups. Univariate association between BG abnormalities and outcomes was further analyzed by multivariate logistic regression analysis after adjusting for confounding variables. Length of stay (LOS) in ICU was analyzed using nonparametric Kaplan-Meier survival analysis. Hazards of discharge from ICU among patients with and without BG variability were further analyzed using Cox proportional hazard analysis after adjusting for confounding variables.

\section{RESULTS}

During the study period, 396 children were admitted; of these 138 patients were excluded ( 3 had diabetic ketoacidosis, 1 had suspected inborn error of metabolism while 134 had incomplete records) from the study. Thus, 258 patients met the inclusion criteria and were analyzed. The median age at admission was 48 months (interquartile range [IQR] 3-144); 191 (74\%) were male. Table 1 describes the baseline characteristics and outcomes of the study cohort. There were no substantial differences between included patients and patients that were excluded because of missing data (data not shown).

Prevalence of blood glucose categories in the study cohort The median BG at admission was $125 \mathrm{mg} / \mathrm{dl}$ (IQR: 95.7-181.2). Figure 1 shows the trends of median maximum and minimum BG values in the first 7 days of ICU stay.

About 139 (53.9\%) patients had isolated hyperglycemia in the first 7 days of ICU stay. Of these, thirty (21.6\%, i.e., 30/139) required insulin for BG control. Exclusive hypoglycemia was seen in nine $(3.5 \%)$ patients; the small number of patients in this group precluded any further analysis. Variability was noted in 76 (29.5\%) patients; $60(23.2 \%)$ had fluctuations in BG levels on the same day. Almost 38 (14.7\%) patients had variable BG levels in the absence of insulin therapy. The remaining $34(13.1 \%)$ patients were normoglycemic (BG levels maintained between 61 and $149 \mathrm{mg} / \mathrm{dl}$ ).

The distribution of admission characteristics among the four BG categories is shown in Table 2. The medical and surgical patients were equally distributed among the four BG groups. The patients in the hypoglycemia group were more malnourished $(P=0.048)$ than the other groups with median

\begin{tabular}{|c|c|}
\hline Variable & Results* \\
\hline Age (months) & $48(3-144)$ \\
\hline \multicolumn{2}{|l|}{ Gender } \\
\hline Male & $191(74)$ \\
\hline Female & $67(26)$ \\
\hline Weight (kg) & $12.2(5-30)$ \\
\hline Weight for age (Z-score) & $-1.5(-2.5--0.7)$ \\
\hline \multicolumn{2}{|l|}{ Type of ICU admission } \\
\hline Medical $^{\#}$ & $141(54.7)$ \\
\hline Liver failure & $36(13.9)$ \\
\hline Tropical illness & $22(8.5)$ \\
\hline Acute flaccid paralysis & $15(5.8)$ \\
\hline Surgical $^{\#}$ & $117(45.3)$ \\
\hline Postoperative $\mathrm{EHBA}^{\dagger}$ & $56(21.7)$ \\
\hline Other abdominal surgeries & $57(22.0)$ \\
\hline Blood sugar at admission to ICU (mg/dl) & $125(95.7-181.2)$ \\
\hline Probability of death by PIM2 at admission (\%) & $2.9(0.4-10.1)$ \\
\hline Presence of infection at admission & $131(50.8)$ \\
\hline Vasoactive agents use & $84(32.6)$ \\
\hline Multiorgan dysfunction syndrome & $106(41.1)$ \\
\hline Length of ICU stay in days & $4(2-9)$ \\
\hline Mortality & $58(22.5)$ \\
\hline \multicolumn{2}{|c|}{$\begin{array}{l}\text { *Values are expressed in median with interquartile range or } n(\%) \text { as } \\
\text { applicable; }{ }^{*} \text { Major diagnoses in the study group; }{ }^{+P a t i e n t s ~ u n d e r w e n t ~} \\
\text { hepatoportoenterostomy for EHBA. ICU: Intensive Care Unit; }\end{array}$} \\
\hline
\end{tabular}


weight for age Z-score of - 2.9 (IQR - 4.6--2.1). However, the number in this group was too small for any definite conclusion. The median probability of death was significantly higher in BG variability group as compared to the normoglycemia group $(7.8 \%$ vs. $0.6 \%, P=0.001)$ and the hyperglycemia group (7.8\% vs. $2.7 \%, P<0.001)$. Similarly, the presence of infection at the time of admission was seen more often in $\mathrm{BG}$ variability than in hyperglycemia $(68.4 \%$ vs. $46.8 \%, P=0.002)$ and normoglycemia group $(68.4 \%$ vs. $29.4 \%, P=0.001)$.

\section{Factors affecting blood glucose abnormalities}

In univariate analysis, presence of infection at admission, use of insulin, probability of death by PIM2 score, and use of vasoactive agents were significantly associated with BG variability $(P<0.05)$. On multivariate regression analysis, only insulin usage (adjusted odds ratio [OR]: 3.6; 95\% confidence

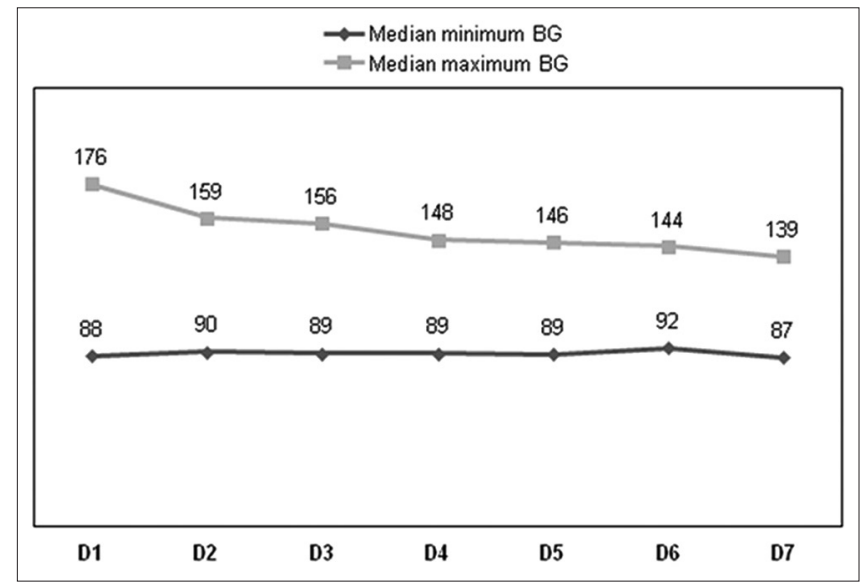

Figure 1: Trends of daily maximum and minimum blood glucose values $(\mathrm{mg} / \mathrm{dl})$ in the first 7 days of Intensive Care Unit stay. interval [CI]: 1.8-7.0) was found to be independently influencing BG variability in the first 7 days of ICU stay. None of these factors significantly affected the occurrence of isolated hyperglycemia.

Association of blood glucose abnormalities with mortality Overall, $58(22.5 \%)$ out of 258 patients died during the study period. Twenty $(14.4 \%)$ patients in hyperglycemia group died as compared to four (11.8\%) in normoglycemia group (OR: 1.3 ; 95\% CI: 0.4-3.9). Patients with isolated BG variability were 5.4 times (95\% CI: $1.7-17.0)$ more likely to die than those with no BG abnormality [Table 3]. Apart from BG groups, medical indications for admission as compared to surgical patients (OR: 13; 95\% CI: 5.2-35.1), PIM2 score (OR: 1.1; 95\% CI: 1.08-1.19), evidence of infection at admission (OR: 13.2; 95\% CI: 5.4-32.4), and presence of MODS (OR: 38.4; 95\% CI: 13.2-111.3) were all associated with increased odds of dying. On multivariate logistic regression analysis, $\mathrm{BG}$ variability failed to retain its effect on mortality (adjusted OR: 1.5; 95\% CI: 0.3-7.1), MODS (adjusted OR: 15.8; 95\% CI: 3.9-63.9), and PIM2 score (adjusted OR: 1.03; 95\% CI: 1.01-1.06) were independent predictors of mortality.

\section{Association of blood glucose abnormalities with multiorgan dysfunction syndrome}

On similar multivariate analysis, we found that among the BG categories, isolated BG variability was independently associated with MODS (adjusted OR: 7.1; 95\% CI: 1.6-31.1). Fifty $(65.8 \%)$ out of 76 patients with variability developed MODS. Other factors that independently influenced the development of MODS were medical admission (adjusted OR: 4.1; 95\% CI: 1.5-11.3), PIM2 score (adjusted OR: 1.1;

Table 2: Distribution of admission characteristics among the four exclusive blood glucose groups

\begin{tabular}{|c|c|c|c|c|}
\hline Variable* & Normoglycemia $(n=34)$ & Hyperglycemia $(n=139)$ & Hypoglycemia $(n=09)$ & BG variability $(n=76)$ \\
\hline Age (months) & $3.7(1.9-114)$ & $48(3.5-156)$ & $6(1.9-90)$ & $60(3.9-153)$ \\
\hline Gender - Male & $23(67.6)$ & $105(75.5)$ & $4(44.4)$ & $59(77.6)$ \\
\hline \multicolumn{5}{|l|}{ Admission type $\mathrm{e}^{\#}$} \\
\hline Medical & $14(41.2)$ & $74(53.2)$ & $6(66.7)$ & $47(62.8)$ \\
\hline Surgical & $20(58.8)$ & $65(46.8)$ & $3(33.3)$ & $29(38.1)$ \\
\hline Weight for age (Z-score) $)^{\#}$ & $-1.3(-2.2--0.4)$ & $-1.5(-2.6--0.5)$ & $-2.9(-4.6--2.1)$ & $-1.4(-2.2--0.7)$ \\
\hline $\begin{array}{l}\text { Probability of death (\%) } \\
\text { by PIM2 score }\end{array}$ & $0.6(0.2-4.3)$ & $2.7(0.4-8.7)$ & $0.6(0.2-5.7)$ & $7.8(1.7-24.5)$ \\
\hline Infection at admission ${ }^{\dagger}$ & $10(29.4)$ & $65(46.8)$ & $4(44.4)$ & $52(68.4)$ \\
\hline
\end{tabular}

*Values are expressed in median with interquartile range or $n(\%)$ as applicable; ${ }^{\sharp} P$ value not significant; ${ }^{\dagger} P<0.05$ between normoglycemia and $\mathrm{BG}$ variability; BG variability and hyperglycemia. BG: Blood glucose; PIM2: Pediatric Index of Mortality-2

Table 3: Clinical outcomes in four exclusive blood glucose categories

\begin{tabular}{llccc}
\hline Outcome & Normoglycemia $(\boldsymbol{n}=\mathbf{3 4})$ & Hyperglycemia $(\boldsymbol{n}=\mathbf{1 3 9})$ & Hypoglycemia* $(\boldsymbol{n}=\mathbf{0 9})$ & BG variability $(\boldsymbol{n}=\mathbf{7 6})$ \\
\hline Mortality, $n(\%)$ & $4(11.8)$ & $20(14.4)$ & $2(22.8)$ & $32(42.1)$ \\
OR $(95 \% \mathrm{CI})$ & Reference category & $1.3(0.4-3.9)$ & - & $5.4(1.7-17.0)$ \\
MODS, $n(\%)$ & $6(17.6)$ & $49(35.3)$ & $1(11.1)$ & $50(65.8)$ \\
OR $(95 \% \mathrm{CI})$ & Reference category & $2.5(0.9-6.5)$ & - & $8.9(3.3-24.5)$ \\
\hline
\end{tabular}

*The number of patients in isolated hypoglycemia group was too small for any meaningful analysis. BG: Blood glucose; OR: Odds ratio; CI: Confidence interval; MODS: Multiple dysfunction syndrome 
95\% CI: 1.04-1.14), and presence of infection at admission (adjusted OR: 6.8; 95\% CI: 2.7-17.4).

\section{Association of blood glucose abnormalities with length of Intensive Care Unit stay}

When the stratifying variable was BG variability, ICU LOS was significantly prolonged in patients who showed variability. Median LOS was 4 days (95\% CI: 3.03-4.97 days) in patients with no BG variability as compared to 11 days (95\% CI: 6.9-15.1 days) in patients showing BG variability; this difference was significant on log-rank test $(P=0.001)$. Results are highlighted graphically using Kaplan-Meier survival curves [Figure 2] where patients who died were censored in each group. On further multivariate Cox proportional hazard analysis, variability in BG level retained its independent predictive ability when adjusted for other confounders such as PIM2, MODS, age, and admission type (medical or surgical). Chances of early discharge were significantly lower in patients who showed BG variability as compared to patients with no BG variability (adjusted hazard ratio [HR]: 0.68; 95\% CI: $0.47-0.98 ; P=0.04$ ) as shown in Figure 2. Early discharge from ICU was also significantly lower in patients with MODS (HR: 0.46 ; 95\% CI: $0.32-0.65 ; P<0.001$ ).

\section{Discussion}

In this retrospective study, we have described the prevalence of different BG abnormalities in a population of critically ill children and their association with adverse clinical outcomes. Glucose disorders were encountered in $86.4 \%$ of our patients, isolated hyperglycemia being most frequent (53.9\%), similar to previous studies in critically ill children. ${ }^{[8,10,16]} \mathrm{BG}$ variability was independently associated with the development of multiple organ dysfunction in critically ill children; the latter is, in turn, an important contributor to mortality.

Globally, approximately $49 \%-72 \%$ of critically ill children experience high BG levels during their ICU stay. $[5,6,8,16,18,19]$

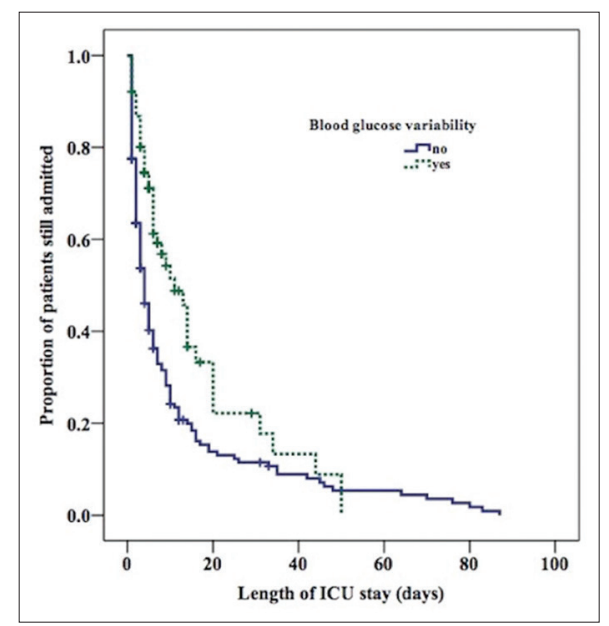

Figure 2: Kaplan-Meier survival analysis for length of Intensive Care Unit stay (adjusted hazard ratio: 0.68; 95\% confidence interval: 0.47-0.98; $P=0.04)$.
Hyperglycemia in acute disease states occurs as a result of an absolute or relative insulin deficiency, insulin resistance, and increased hepatic gluconeogenesis. This may be beneficial in the initial phase of illness; however, with continued stress, high BG levels increase the oxidative stress, thereby causing free radical injury to cells. Hyperglycemia is also prothrombotic and proinflammatory and decreases neutrophil phagocytosis, thus promoting infection.

We did not find any significant association between isolated hyperglycemia and mortality or MODS. Multivariate analysis also did not reveal any significant association between isolated hyperglycemia and length of ICU stay. This finding is in contradiction to other studies ${ }^{[5,6,8,16,18,20]}$ and could be because of the difference in clinical profile of the patients presenting to our hospital. Overall, the single largest diagnostic group was postoperative cases of extrahepatic biliary atresia (21.7\%). Further, not all other authors have studied exclusive BG groups for analysis, and the deleterious effects could be because of variability in BG levels rather than hyperglycemia itself. Several studies have not taken severity of illness into account. ${ }^{[6,16,20]}$ Klein et al. have shown no independent association of hyperglycemia on mortality and morbidity when disease severity was taken into account. ${ }^{[21]}$

The prevalence of BG variability in our study cohort was high $(29.5 \%)$ as compared to other studies in literature that used the same definition for variability; ${ }^{[8,10]}$ incidence decreased to $14.7 \%$ in the absence of insulin. The adverse effects of variability are attributable to increase in endothelial damage and apoptosis as a result of fluctuations in BG levels. ${ }^{[15,22]}$

We found a significant association between BG variability and mortality in our patients on univariate analysis. However, this association was lost when MODS, severity of illness, presence of infection at admission, and type of admission were taken into account. We found increased ICU stay in patients showing BG variability as compared to those without it as also reported by earlier studies..$^{[8,10,16,22]}$ The lack of association with mortality as found in these studies may be explained by overlap of patients between the BG categories in these studies. Unfortunately, we are unable to refute or support this hypothesis due to extremely small number of isolated hypoglycemia in our study. We did find $\mathrm{BG}$ variability to be an independent predictor of MODS on multivariate analysis. To the best of our knowledge, only one previous study has found a significant relationship between BG variability and MODS..$^{[10]}$

The frequency of isolated hypoglycemia in our patients was low $(3.5 \%)$ which is similar to that reported by Hirshberg. ${ }^{[8]}$ Studies done in various pediatric ICUs have reported 7\%-9.7\% incidence of spontaneous hypoglycemia. ${ }^{[2,3,8,23]}$ The development of hypoglycemia in critical illness is attributed to catabolic state with impairment of carbohydrate metabolism leading to increased utilization and decreased production of glucose. In addition, inadequate nutrition and use of insulin to treat hyperglycemia contributes to occurrence of hypoglycemia. 
Among the factors affecting BG abnormalities, we found insulin to be an independent predictor of BG variability. In general, almost one-quarter of our patients $(22.8 \%)$ received insulin for the management of hyperglycemia though we did not have any fixed protocol for BG monitoring and insulin titration. Hypoglycemia has been reported often in relation to tight glycemic control; however, some recent studies have reported the use of protocol-based insulin infusions without an increase in adverse events (i.e., hypoglycemia). ${ }^{[23-25]}$ Till now, very few studies have evaluated factors affecting BG abnormalities (i.e., hyperglycemia and BG variability). Bhutia et al. found the use of steroids to be associated with increased hyperglycemia ${ }^{[10]}$ and Preissig and Rigby found that organ failure was associated with the occurrence of hyperglycemia. ${ }^{[19]}$

\section{Strengths}

Our study adds to the existing limited pediatric literature regarding alterations in BG levels in diverse populations of critically ill children. We believe that exclusive BG categories should be used for analysis, as in only one other previous study. The severity of illness was discerned by PIM2 scoring system, which does not include BG level as compared to Pediatric Risk of Mortality III score. This is also among the few studies reporting the factors affecting alterations in BG homeostasis.

\section{Limitations}

Our study has inherent limitation of being a retrospective study. Patients with BG abnormalities were more frequently monitored as compared to those with normal BG. The absence of a fixed monitoring protocol for BG management might have led to missed episodes of abnormal BG levels. In addition, we have not assessed the effect of glucose intake on BG abnormalities.

\section{Conclusion}

Alterations in BG levels are frequently encountered in critically ill children. BG variability is independently associated with the development of MODS and increased ICU LOS. Insulin use is independently associated with the occurrence of BG variability.

\section{Acknowledgments}

Dr. Sahul Bharti provided expert guidance for statistical analysis, and we are grateful for his help.

\section{Financial support and sponsorship}

Nil.

\section{Conflicts of interest}

There are no conflicts of interest.

\section{RefERENCES}

1. Lodha R, Bhutia TD, Kabra SK, Thukral A. Day 1 blood glucose and outcome in critically ill children. Indian Pediatr 2009;46:809-10.

2. Faustino EV, Bogue CW. Relationship between hypoglycemia and mortality in critically ill children. Pediatr Crit Care Med 2010;11:690-8.

3. Faustino EV, Hirshberg EL, Bogue CW. Hypoglycemia in critically ill children. J Diabetes Sci Technol 2012;6:48-57.

4. van den Berghe G, Wouters P, Weekers F, Verwaest C, Bruyninckx F, Schetz M, et al. Intensive insulin therapy in critically ill patients. N Engl J Med 2001;345:1359-67.

5. Srinivasan V, Spinella PC, Drott HR, Roth CL, Helfaer MA, Nadkarni V. Association of timing, duration, and intensity of hyperglycemia with Intensive Care Unit mortality in critically ill children. Pediatr Crit Care Med 2004;5:329-36.

6. Faustino EV, Apkon M. Persistent hyperglycemia in critically ill children. J Pediatr 2005;146:30-4.

7. Mikos B, Barkai L. Hyperglycemia and mortality in critically ill children. Orv Hetil 2007;148:683-8.

8. Hirshberg E, Larsen G, Van Duker H. Alterations in glucose homeostasis in the pediatric Intensive Care Unit: Hyperglycemia and glucose variability are associated with increased mortality and morbidity. Pediatr Crit Care Med 2008;9:361-6.

9. Wintergerst KA, Foster MB, Sullivan JE, Woods CR. Association of hyperglycemia, glucocorticoids, and insulin use with morbidity and mortality in the pediatric Intensive Care Unit. J Diabetes Sci Technol 2012;6:5-14

10. Bhutia TD, Lodha R, Kabra SK. Abnormalities in glucose homeostasis in critically ill children. Pediatr Crit Care Med 2013;14:e16-25.

11. Agus MS, Steil GM, Wypij D, Costello JM, Laussen PC, Langer M, et al. Tight glycemic control versus standard care after pediatric cardiac surgery. N Engl J Med 2012;367:1208-19.

12. Macrae D, Grieve R, Allen E, Sadique Z, Morris K, Pappachan J, et al. A randomized trial of hyperglycemic control in pediatric intensive care. N Engl J Med 2014;370:107-18.

13. Egi M, Bellomo R, Stachowski E, French CJ, Hart G. Variability of blood glucose concentration and short-term mortality in critically ill patients. Anesthesiology 2006;105:244-52.

14. Ali NA, O’Brien JM Jr., Dungan K, Phillips G, Marsh CB, Lemeshow S, et al. Glucose variability and mortality in patients with sepsis. Crit Care Med 2008;36:2316-21.

15. Siegelaar SE, Holleman F, Hoekstra JB, DeVries JH. Glucose variability; does it matter? Endocr Rev 2010;31:171-82.

16. Wintergerst KA, Buckingham B, Gandrud L, Wong BJ, Kache S, Wilson DM. Association of hypoglycemia, hyperglycemia, and glucose variability with morbidity and death in the pediatric Intensive Care Unit. Pediatrics 2006;118:173-9.

17. Patki VK, Chougule SB. Hyperglycemia in critically ill children. Indian J Crit Care Med 2014;18:8-13.

18. Yung M, Wilkins B, Norton L, Slater A; Paediatric Study Group; Australian and New Zealand Intensive Care Society. Glucose control, organ failure, and mortality in pediatric intensive care. Pediatr Crit Care Med 2008;9:147-52.

19. Preissig CM, Rigby MR. Pediatric critical illness hyperglycemia: Risk factors associated with development and severity of hyperglycemia in critically ill children. J Pediatr 2009;155:734-9.

20. Yates AR, Dyke PC $2^{\text {nd }}$, Taeed R, Hoffman TM, Hayes J, Feltes TF, et al. Hyperglycemia is a marker for poor outcome in the postoperative pediatric cardiac patient. Pediatr Crit Care Med 2006;7:351-5.

21. Klein GW, Hojsak JM, Schmeidler J, Rapaport R. Hyperglycemia and outcome in the pediatric Intensive Care Unit. J Pediatr 2008;153:379-84.

22. Rake AJ, Srinivasan V, Nadkarni V, Kaptan R, Newth CJ. Glucose variability and survival in critically ill children: Allostasis or harm? Pediatr Crit Care Med 2010;11:707-12.

23. Preissig CM, Hansen I, Roerig PL, Rigby MR. A protocolized approach to identify and manage hyperglycemia in a pediatric Critical Care Unit. Pediatr Crit Care Med 2008;9:581-8.

24. Garcia Branco R, Tasker RC, Ramos Garcia PC, Piva JP, Dias Xavier L. Glycemic control and insulin therapy in sepsis and critical illness. J Pediatr (Rio J) 2007;83 5 Suppl: S128-36.

25. Faraon-Pogaceanu C, Banasiak KJ, Hirshberg EL, Faustino EV. Comparison of the effectiveness and safety of two insulin infusion protocols in the management of hyperglycemia in critically ill children. Pediatr Crit Care Med 2010;11:741-9. 